\title{
DYNAMIC MOTION OF A CONICAL FRUSTUM OVER A ROUGH HORIZONTAL PLANE
}

\author{
Ioannis Stefanou ${ }^{a}$, Ioannis Vardoulakis $\dagger$ and Anastasios Mavraganis ${ }^{b}$ \\ ${ }^{a}$ Department of Applied Mathematics and Physics \\ National Technical University of Athens \\ P.O.BOX 15780, Athens, Greece \\ e-mail: istefanou@mechan.ntua.gr, \\ web page: http://geolab.mechan.ntua.gr/ \\ (Corresponding author ${ }^{1}$ ) \\ ${ }^{\mathrm{b}}$ Department of Applied Mathematics and Physics \\ National Technical University of Athens \\ P.O.BOX 15780, Athens, Greece \\ e-mail: tamavra@central.ntua.gr
}

\section{Abstract:}

An analytical and numerical study of the dynamic motion of a conical frustum over a planar surface is presented resulting to a non-linear system of ordinary differential equations. Wobbling and rocking components of motion are discussed in detail concluding that, in general, the former component dominates the latter. For small inclination angles an asymptotic approximation of the angular velocities is possible, revealing the main characteristics of wobbling motion and its differences from rocking. Connection is made of the analysis with the behavior of the ancient classical columns, whose three dimensional dynamic response challenges the accuracy of the two dimensional models, usually applied in practice. The consideration of such discrete-blocky systems can benefit from the present study, through qualitative results and benchmarks for more complicated numerical methods, like the Distinct Element Method.

Keywords: Conical frustum; Dynamic motion; Wobbling; Rocking; Ancient classical columns.

\footnotetext{
${ }^{1}$ Corresponding Author:

Ioannis Stefanou

Department of Applied Mathematics and Physics

National Technical University of Athens

P.O.BOX 15780, Athens, Greece

e-mail: istefanou@mechan.ntua.gr,

web page: http://geolab.mechan.ntua.gr/

tel: +306977212890

fax: +302106722873

$\dagger$ Deceased.
} 


\section{INTRODUCTION}

The non-holonomic problem of a symmetric body by revolution, rolling on a planar surface, was first formulated by Routh in 1868 [1]. Since then, a significant number of papers appeared on this subject, focusing mostly on the study of the motion of a thin disk on a horizontal plane. The elaboration of the problem of the thin disk is presented in most classical textbooks of Dynamics [2, 3, 4, 5] providing to the readers a typical example of nonholonomic motion. Noticing the early works of Appell [6] in 1900 (cf. also Korteweg [7], 1900) and Gallop [8] in 1904, where analytic solutions are given in terms of Gauss hypergeometric and Legendre functions, we pass to the corps of papers of the current decade. The papers of O'Reilly [9] (1996), Kuleshov [10] (2001), Paris and Zhang [11] (2002), Kessler and O'Reilly [12] (2002), Borisov et al. [13] (2003), Le Saux et al. [14] (2005) provide a deep insight to the dynamic behavior of the thin disc. Equally important for the present study are also the papers of Koh and Mustafa [15] (1990) and Batista [16] (2006), which discuss the motion of a disc of finite thickness on a planar surface. In the latter papers the equations of motion of a cylindrical drum are derived and numerical simulations are performed.

In the present paper we deal with the case of a conical frustum, rolling on a rough horizontal surface. Using for the description of motion the Lagrange formulation, we distinguish between the wobbling and the rocking of the frustum and comment extensively on these components of motion. Stability analysis reveals the pure three dimensional character of the motion, while further approximations of the angular velocities under small inclination angles are elaborated to examine the main characteristics of the motion of the frustum. Finally, an attempt is made to interpret the dynamical behavior of ancient classical columns considering them as conical frustums with slightly different radii.

\section{EQUATIONS OF MOTION OF A CONICAL FRUSTUM ON A ROUGH HORIZONTAL PLANE}
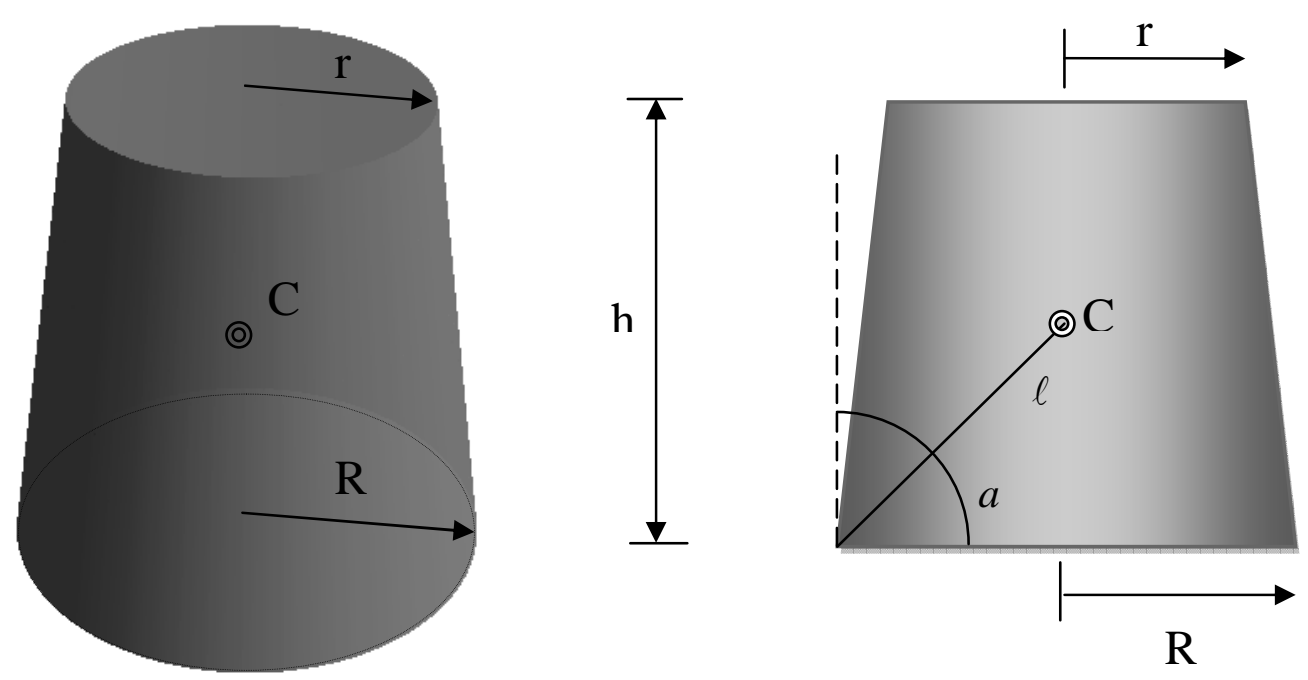

Figure 1. The conical frustum: $3 D$ and $2 D$ view. 
The formulation of the problem is based on the following assumptions:

a. The body is a homogeneous, rigid conical frustum.

b. The contact with the horizontal plane is assumed punctual. Notice that Kessler and O'Reilly [12] introduced a contact moment for simulating a 'flat' contact. This additional complication is not considered here, because rolling friction is disregarded.

c. At any given time the body is in contact with its horizontal planar base and only smooth transitions in time are considered.

\subsection{Formulation of the system}

The position of the body in the inertial frame $O(X Y Z)$ is determined by the coordinates of the contact point $P\left(X_{P}, Y_{P}\right)$ and by the Euler angles $(\varphi, \theta, \psi)$, where $\varphi$ is the precession angle, $\theta$ the inclination (nutation) angle and $\psi$ the rotation about $\zeta$-axis (Figure 2). For $\theta=0$ the frustum comes into contact with the horizontal plane by whole base. Hence, the motion is restricted in the interval $\theta \in[0, \pi / 2]$.

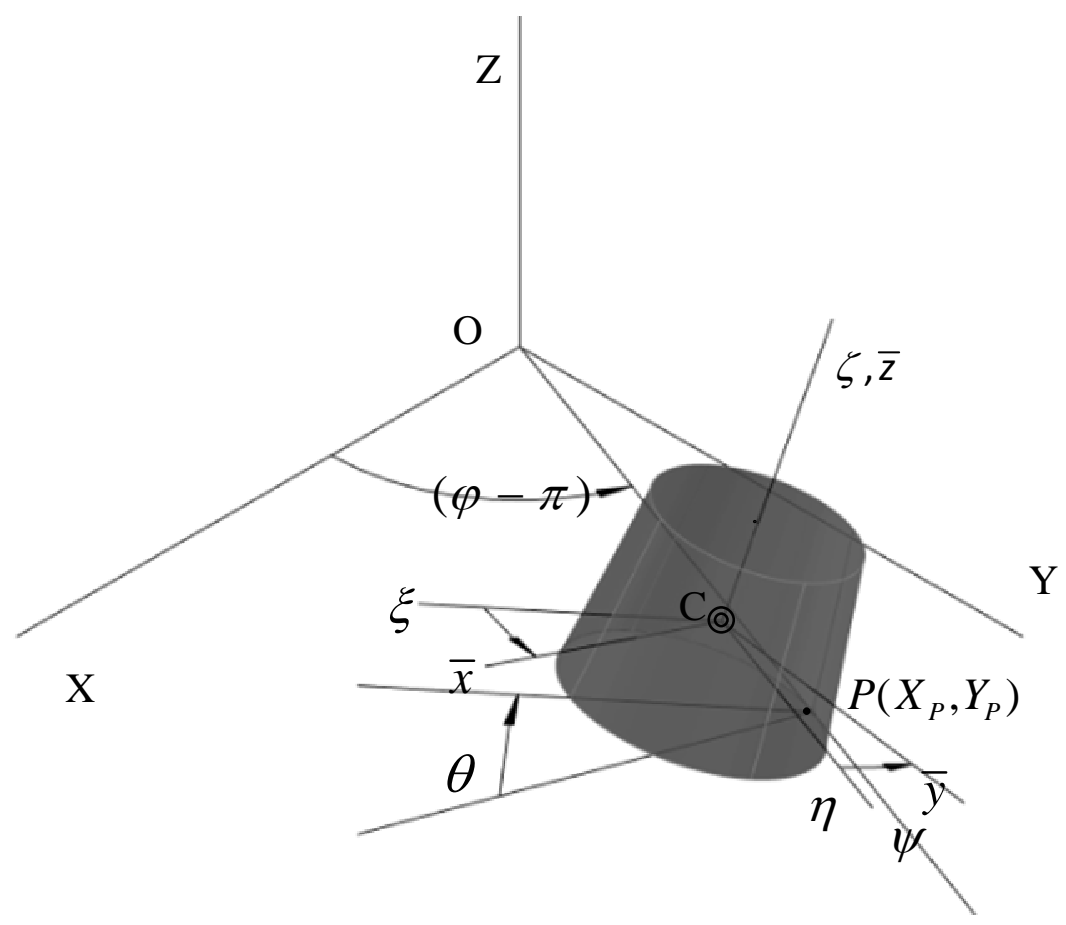

Figure 2. Coordinate systems and Euler angles. $O(X, Y, Z)$ is the inertial frame, $P\left(X_{P}, Y_{P}\right)$ the contact point with the horizontal plane, $\theta$ the inclination angle, $\varphi$ the precession angle and $\psi$ the rotation about $\zeta$-axis.

If the frustum rolls without sliding then the velocity of the contact point $P\left(X_{P}, Y_{P}\right)$ is:

$$
V_{P}=-R \dot{\psi}
$$

where $R$ is the radius of the base of the drum. Applying the Frobenius criterion, it may be easily proven that constraint (1) is non-holonomic.

Ground accelerations can also be considered by introducing the additional inertia terms:

$$
\dot{X}_{P}+R \dot{\psi} \cos \varphi=\alpha_{X}^{g r} \dot{u}_{g r}(t) \quad, \quad \dot{Y}_{P}+R \dot{\psi} \sin \varphi=\alpha_{Y}^{g r} \dot{u}_{g r}(t)
$$


where $\alpha_{X}^{g r}, \alpha_{Y}^{g r}$ are two scalar quantities, constant in time, that express the direction of the ground acceleration $\ddot{u}_{g r}(t)$.

Given the frictional law of the materials in contact (eg. Coulomb friction), the estimation of the sliding velocity is feasible by combining the velocity of the point $P$, regarded as a point of the frustum, with the frictional forces developed at the contact. However, this formulation extends the limits and the scope of the present paper and it will not be pursued further hereafter. Numerical and parametric studies that include sliding are, of course, important for practical applications, as they supply quantitative information to be used for design purposes, but add little to the qualitative understanding of the basic dynamics of the system.

The angular velocity components of the body relative to $C(\xi \eta \zeta)$ are:

$$
\begin{aligned}
& \omega_{\xi}=\dot{\theta} \\
& \omega_{\eta}=\dot{\varphi} \sin \theta \\
& \omega_{\zeta}=\dot{\varphi} \cos \theta+\dot{\psi}
\end{aligned}
$$

whilst the components relative to the central principal axes system $C(\bar{x} \bar{y} \bar{z})$ are:

$$
\begin{aligned}
& \omega_{\bar{x}}=\dot{\theta} \cos \psi+\dot{\varphi} \sin \theta \sin \psi \\
& \omega_{\bar{y}}=-\dot{\theta} \sin \psi+\dot{\varphi} \sin \theta \cos \psi \\
& \omega_{\bar{z}}=\dot{\varphi} \cos \theta+\dot{\psi}
\end{aligned}
$$

Notice the coincidence of $\omega_{\bar{z}}$ and $\omega_{\zeta}$, because $\bar{z}=\zeta$.

The coordinates of the center mass of the conical frustum in $O(X Y Z)$ are given in terms of the contact point coordinates $P\left(X_{P}, Y_{P}\right)$, by the following relations:

$$
\begin{aligned}
X_{c} & =X_{P}-\ell \cos (a+\theta) \sin \varphi \\
Y_{c} & =Y_{P}+\ell \cos (a+\theta) \cos \varphi \\
Z_{c} & =\ell \sin (a+\theta)
\end{aligned}
$$

where $\ell=\sqrt{R^{2}+\frac{1}{4} k_{1}^{2} h^{2}}, \tan a=\frac{k_{1} h}{2 R}, k_{1}=2 \frac{\bar{z}_{c m}}{h}, \bar{z}_{c m}=\frac{3 \beta^{2}+2 \beta+1}{\beta^{2}+\beta+1} \frac{h}{4}, \beta=\frac{r}{R}$,

$r$ and $R$ are respectively the radii of the upper and lower rim of the conical frustum and $h$ is its height (Figure 1).

The velocity $\mathbf{V}_{C}$ of the center of the mass of the frustum yields:

$$
\begin{aligned}
\dot{X}_{C} & =\dot{X}_{P}+\ell \dot{\theta} \sin (a+\theta) \sin \varphi-\ell \dot{\varphi} \cos (a+\theta) \cos \varphi \\
\dot{Y}_{C} & =\dot{Y}_{P}-\ell \dot{\theta} \sin (a+\theta) \cos \varphi-\ell \dot{\varphi} \cos (a+\theta) \sin \varphi \\
\dot{Z}_{C} & =\ell \dot{\theta} \cos (a+\theta)
\end{aligned}
$$

\subsection{Dynamic equations of motion}

The kinetic and the potential energy of the drum are: 


$$
\begin{aligned}
& T=\frac{1}{2} m \mathbf{V}_{C}^{2}+\frac{1}{2} \boldsymbol{\omega}^{\mathbf{T}} \mathbf{I}_{C} \boldsymbol{\omega} \\
& V=m g z_{C}
\end{aligned}
$$

where $\mathbf{I}_{C}$ is the inertia tensor relative to $C(\bar{x} \bar{y} \bar{z})$ :

$$
\begin{aligned}
& \mathbf{I}_{C}=\mathbf{I}_{P}-\left(\begin{array}{ccc}
\frac{1}{4} k_{1} m h^{2}+m R^{2} & 0 & 0 \\
0 & \frac{1}{4} k_{1} m h^{2}+m R^{2} & 0 \\
0 & 0 & m R^{2}
\end{array}\right), \mathbf{I}_{P}=\left(\begin{array}{ccc}
I_{\bar{x}}^{P} & 0 & 0 \\
0 & I_{\bar{y}}^{P} & 0 \\
0 & 0 & I_{\bar{z}}^{P}
\end{array}\right), \\
& I_{\bar{z}}^{P}=\frac{1}{2}\left(k_{3}+2\right) m R^{2}, I_{\bar{x}}^{P}=I_{\bar{y}}^{P}=\frac{1}{4}\left(k_{3}+4\right) m R^{2}+\frac{1}{12}\left(k_{2}+3 k_{1}^{2}\right) m h^{2} \\
& k_{2}=\frac{9}{20} \frac{\beta^{4}+4 \beta^{3}+10 \beta^{2}+4 \beta+1}{\left(\beta^{2}+\beta+1\right)^{2}} \text { and } k_{3}=\frac{3}{5} \frac{\beta^{4}+\beta^{3}+\beta^{2}+\beta+1}{\beta^{2}+\beta+1}
\end{aligned}
$$

The inertia tensor $\mathbf{I}_{P}$ expresses the inertia moments of the body at the contact point $P$. For cylindrical drums it holds $k_{1}=k_{2}=k_{3}=\beta=1$.

Introducing the generalized coordinates $q_{1}=\varphi, q_{2}=\theta, q_{3}=\psi, q_{4}=X_{P}$ and $q_{5}=Y_{P}$ the general form of the Lagrange equations for non-holonomic systems are:

$$
\frac{d}{d t}\left[\frac{\partial(T-V)}{\partial \dot{q}_{i}}\right]-\frac{\partial(T-V)}{\partial q_{i}}-\sum_{j=1}^{2} \lambda_{j} B_{j i}=0
$$

With $\lambda_{i}$ we denote the Lagrange multipliers, while:

$$
B_{j i}=\frac{\partial\{\text { non-holonomic constraint equation ' } j '\}}{\partial \dot{q}_{i}}, \text { resulting to: }\left\{B_{j i}\right\}=\left(\begin{array}{lllll}
1 & 0 & 0 & 0 & R \cos \phi \\
0 & 1 & 0 & 0 & R \sin \phi
\end{array}\right) \text {. }
$$

For convenience we introduce the following dimensionless quantities:

$$
\begin{gathered}
\hat{h}=\frac{h}{R}, \quad \tau=t \sqrt{\frac{g}{R}}, \quad \hat{X}=\frac{X_{P}}{R}, \quad \hat{Y}=\frac{Y_{P}}{R}, \quad \hat{u}_{g r}=\frac{u_{g r}}{R}, \quad(.)^{\prime} \equiv \frac{d(.)}{d \tau}, \\
\hat{I}_{k}=\frac{I_{k}}{m R^{2}}, \quad \hat{\omega}_{k}=\omega_{k} \sqrt{\frac{R}{g}}, \quad \hat{T}=\frac{T}{m g R}, \quad \hat{V}=\frac{V}{m g R}, \quad \hat{E}=\frac{E}{m g R}
\end{gathered}
$$

where $g$ is the acceleration of gravity and $E$ the total energy of the system. According to Eq.(8), the equations of motion are written in matrix notation:

$$
\mathbf{A} \cdot \mathbf{U}=\mathbf{B}
$$

where $\mathbf{U}=\left\{\varphi^{\prime \prime}, \theta^{\prime \prime}, \psi^{\prime \prime}, \lambda_{1}, \lambda_{2}, \hat{x}^{\prime \prime}, \hat{y}^{\prime \prime}\right\}$ and $\mathbf{A}, \mathbf{B}$ are matrices given in Appendix A.

The determinant of matrix $\mathbf{A}$ is: 


$$
\begin{aligned}
\operatorname{det}(\mathbf{A}) & =\frac{1}{4} \hat{I}_{\bar{y}}^{P}\left[4\left(\hat{I}_{\bar{y}}^{P}-1\right) \hat{I}_{\bar{z}}^{P}-\hat{h}^{2} k_{1}^{2}\right] \sin ^{2} \theta \\
& =\frac{1}{288}\left[\left(3 k_{1}^{2}+k_{2}\right) \hat{h}^{2}+3\left(k_{3}+4\right)\right]\left[\left[3 k_{3} k_{1}^{2}+k_{2}\left(k_{3}+2\right)\right] \hat{h}^{2}+3\left(k_{3}+2\right)\right] \sin ^{2} \theta>0
\end{aligned}
$$

for $\theta>0$.

For $\theta=0, \operatorname{det}(\mathbf{A})=0$ and the system of Eqs.(10) is singular. In the limit of $\theta \rightarrow 0^{+}$, a smooth full contact of the drum with the horizontal plane is reached. This collision is not trivial in the sense that the impact is taking place between surfaces in the three dimensional space.

Consequently, the impact involves impulse reaction forces and torques, including torsion. The hypotheses usually made for the dynamics and the contact point just after the impact $[9,17$, 18] permit the application of the angular momentum principle and the calculation of a unique restitution coefficient. However, these hypotheses are not straightforward, because of the spin of the body at the instant of the impact, which may influence the dynamics of the collision even in the frictionless limit. Besides, as it is will be shown in the next sections, impact happens only when a special initial condition is satisfied.

Equations (10) describe the three dimensional motion of a frustum over a shaking rough horizontal plane. These equations are non-linear and for $\theta \neq 0$ they become:

$$
\begin{aligned}
\varphi^{\prime \prime} & =\left[g_{11}(\theta) \varphi^{\prime}+g_{13}(\theta) \psi^{\prime}\right] \theta^{\prime}+g_{14}(\theta) \hat{u}_{g r}^{\prime \prime} \\
\theta^{\prime \prime} & =\left[g_{21}(\theta) \varphi^{\prime}+g_{23}(\theta) \psi^{\prime}\right] \varphi^{\prime}+g_{22}(\theta)+g_{24}(\theta) \hat{u}_{g r}^{\prime \prime} \\
\psi^{\prime \prime} & =\left[g_{31}(\theta) \varphi^{\prime}+g_{33}(\theta) \psi^{\prime}\right] \theta^{\prime}+g_{34}(\theta) \hat{u}_{g r}^{\prime \prime} \\
\hat{X}^{\prime \prime} & =\varphi^{\prime} \psi^{\prime} \sin \varphi-\psi^{\prime \prime} \cos \varphi+\alpha_{1}^{g r} \hat{u}_{g r}^{\prime \prime} \\
\hat{Y}^{\prime \prime} & =-\varphi^{\prime} \psi^{\prime} \cos \varphi-\psi^{\prime \prime} \sin \varphi+\alpha_{2}^{g r} \hat{u}_{g r}^{\prime \prime}
\end{aligned}
$$

where:

$$
\begin{aligned}
g_{11}= & \frac{2\left(\hat{I}_{\bar{z}}^{P}-1\right)\left(2 \cot \theta \hat{I}_{\bar{z}}^{P}-\hat{h} k_{1}\right)}{4\left(\hat{I}_{\bar{y}}^{P}-1\right) \hat{I}_{\bar{z}}^{P}-\hat{h}^{2} k_{1}^{2}}-2 \cot \theta ; g_{13}=\frac{4 \csc \theta\left(\hat{I}_{\bar{z}}^{P}-1\right) \hat{I}_{\bar{z}}^{P}}{4\left(\hat{I}_{\bar{y}}^{P}-1\right) \hat{I}_{\bar{z}}^{P}-\hat{h}^{2} k_{1}^{2}} \\
g_{21}= & \frac{\left(\hat{I}_{\bar{y}}^{P}-\hat{I}_{\bar{z}}^{P}-1\right) \sin 2 \theta-\hat{h} k_{1} \cos 2 \theta}{2 \hat{I}_{\bar{y}}^{P}} ; g_{22}=\frac{\hat{h} k_{1} \sin \theta-2 \cos \theta}{2 \hat{I}_{\bar{y}}^{P}} ; g_{23}=-\frac{2\left(\hat{I}_{\bar{z}}^{P}+1\right) \sin \theta+\hat{h} k_{1} \cos \theta}{2 \hat{I}_{\bar{y}}^{P}} ; \\
g_{31}= & \frac{\left[4 \hat{h} k_{1} \cos \theta-4\left(\hat{I}_{\bar{y}}^{P}-\hat{I}_{\bar{z}}^{P}-1\right) \sin \theta\right]\left(\hat{I}_{\bar{z}}^{P}-1\right)+2\left(4 \hat{I}_{\bar{y}}^{P} \hat{I}_{\bar{z}}^{P}-2 \hat{I}_{\bar{z}}^{P 2}-2 \hat{I}_{\bar{z}}^{P}-\hat{h}^{2} k_{1}^{2}\right) \csc \theta}{4\left(\hat{I}_{\bar{y}}^{P}-1\right) \hat{I}_{\bar{z}}^{P}-\hat{h}^{2} k_{1}^{2}} ; \\
g_{33}= & -\frac{2\left(\hat{I}_{\bar{z}}^{P}-1\right)\left(2 \hat{I}_{\bar{z}}^{P} \cot \theta-\hat{h} k_{1}\right)}{4\left(\hat{I}_{\bar{y}}^{P}-1\right) \hat{I}_{\bar{z}}^{P}-\hat{h}^{2} k_{1}^{2}} ; \\
g_{14}= & \frac{2 \hat{h}_{1}\left(\hat{I}_{\bar{z}}^{P}-1\right) \csc \theta}{\hat{h}^{2} k_{1}^{2}-4\left(\hat{I}_{\bar{y}}^{P}-1\right) \hat{I}_{\bar{z}}^{P}}\left(\alpha_{1}^{g r} \cos \varphi+\alpha_{2}^{g r} \sin \varphi\right) ; \quad g_{24}=\frac{2 \sin \theta+\hat{h} k_{1} \cos \theta}{2 \hat{I}_{\bar{y}}^{P}}\left(-\alpha_{1}^{g r} \sin \varphi+\alpha_{2}^{g r} \cos \varphi\right) ; \\
& 4\left(\hat{I}_{\bar{y}}^{P}-1\right)+2\left(\hat{I}_{\bar{z}}^{P}-1\right) \cot \theta-\hat{h}^{2} k_{1}^{2} \\
g_{34}= & \left.\frac{4\left(\hat{I}_{\bar{y}}^{P}-1\right) \hat{I}_{\bar{z}}^{P}-\hat{h}^{2} k_{1}^{2}}{g r} \cos \varphi+\alpha_{2}^{g r} \sin \varphi\right)
\end{aligned}
$$


We observe that the $\cot \theta$ and $\csc \theta$ terms of Eq.(12) result into a singularity for $\varphi^{\prime \prime}$ and $\psi^{\prime \prime}$ that is carried over $\theta^{\prime \prime}$. The unphysical unlimited angular accelerations predicted by this model for $\theta \rightarrow 0^{+}$imply that the assumption we made here of frictionless contact is too strong for this limit, since "large" angular accelerations would lead to strong tangential reaction forces. These forces would in turn violate the imposed non-sliding constraint. In Figure 3 we plot: a) the normalized inclination angle and b) the mobilized friction coefficient as functions of time. The mobilized friction coefficient is defined here as the ratio of the magnitude of the total tangential force over the magnitude of the normal force developed at the contact point. From this figure follows that in certain occasions a short duration slip would occur leading to energy losses, because of the increased mobilized friction coefficient. The energy dissipated in these short time intervals of sliding should lead to a high-frequency stick and slip mechanism ${ }^{2}$ depending on the assumed friction law [19], that will result eventually to a practically smooth collisionless contact. This remark enforces the conclusion that in the problem at hand, and in general, impact is unattainable.

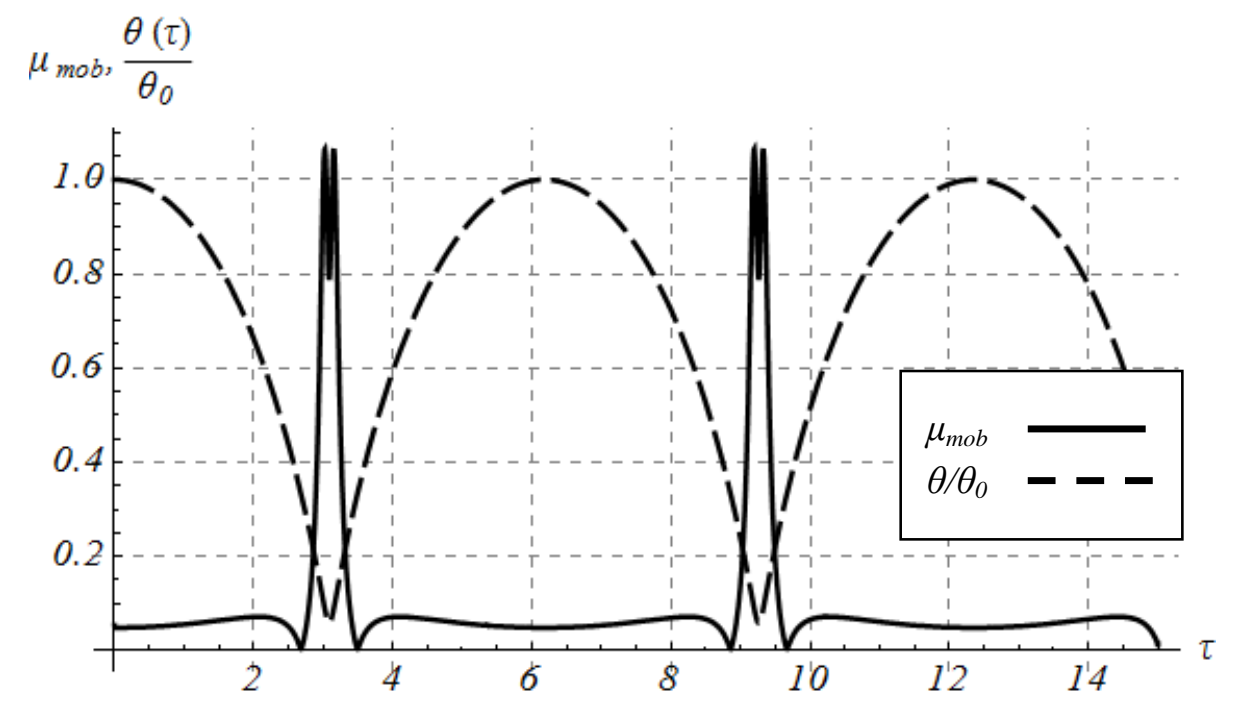

Figure 3. Mobilized friction coefficient and normalized inclination angle of the column presented in Figure 4. The energy dissipated during the short time intervals of sliding (peaks of the mobilized friction coefficient) generally results to a practically smooth collisionless contact.

For zero ground accelerations the system is autonomous and the Jacobi's integral exists and reduces to the total energy of the system [20]:

$$
\begin{aligned}
\hat{E}= & \frac{1}{2} \hat{I}_{\bar{y}}^{P} \theta^{\prime 2}+\frac{1}{2}\left[\hat{I}_{\bar{z}}^{P} \cos ^{2} \theta+\sin \theta\left[\left(\hat{I}_{\bar{y}}^{P}-1\right) \sin \theta-\hat{h} k_{1} \cos \theta\right]\right] \varphi^{\prime 2}+\frac{1}{2} \hat{I}_{\bar{z}}^{P} \psi^{\prime 2} \\
& +\left(\hat{I}_{\bar{z}}^{P} \cos \theta-\frac{1}{2} \hat{h} k_{1} \sin \theta\right) \varphi^{\prime} \psi^{\prime}+\frac{1}{2} e \\
= & \frac{1}{2} \hat{I}_{\bar{y}}^{P} \omega_{\xi}^{2}+\frac{1}{2}\left(\hat{I}_{\bar{y}}^{P}-1\right) \omega_{\eta}^{2}+\frac{1}{2} \hat{I}_{\bar{z}}^{P} \omega_{\zeta}^{2}-\frac{1}{2} \hat{h} k_{1} \omega_{\eta} \omega_{\zeta}+\frac{1}{2} e
\end{aligned}
$$

In the general case of non-autonomous systems, as the one described above, the equations of motion can only be numerically integrated. The numerical scheme that was applied here was

\footnotetext{
${ }^{2}$ In the experiment of a "wobbling" coin on a table this is sensed by a high frequency noise that is produced towards the last phase of the motion.
} 
compared and validated by extending the analytical solution proposed for cylindrical drums in [17] to conical frustums. The validation is presented in Appendix B.

\section{THREE DIMENSIONAL CHARACTER OF THE MOTION}

The initial conditions define the trajectory of the frustum on the horizontal plane. For certain conditions this trajectory may be a circle, a line or even a point. In the particular case, where the trace of the contact point is stationary and $\psi_{0}^{\prime}=\phi_{0}^{\prime}=0$, the motion degenerates into a twodimensional rocking in the vertical plane. In this case the behavior of the system is described by the following equation only:

$$
\theta^{\prime \prime}=\frac{\hat{h} k_{1} \sin \theta-2 \cos \theta}{2 \hat{I}_{\bar{y}}^{P}}
$$

Rocking is described by Eq.(14) with the additional assumption that when the block turns back to the vertical position $(\theta=0)$ it collides with the horizontal plane (i.e. it does not crossover). Then, the contact point changes abruptly to the other side of the circular base, the angular momentum is preserved and the rotation continues about the new contact point until a maximum inclination angle is reached. Therefore, rocking could be seen as a particular case of the general three dimensional motion (Eqs.12) between the time interval of two full contacts with the horizontal plane. This consideration makes the stability analysis of rocking meaningful for our study.

\subsection{Linear stability analysis of rocking}

Introducing the following small perturbations of the dependent variables,

$$
\varphi \rightarrow \varphi_{0}+\tilde{\varphi}(\tau) \quad, \quad \theta \rightarrow \theta(\tau)+\tilde{\theta}(\tau) \quad, \quad \psi \rightarrow \psi_{0}+\tilde{\psi}(\tau)
$$

and by linearizing Eqs.(12), we obtain:

$$
\begin{aligned}
\tilde{\varphi}^{\prime \prime} & =\left[g_{11} \tilde{\varphi}^{\prime}+g_{13} \tilde{\psi}^{\prime}\right] \theta^{\prime} \\
\tilde{\theta}^{\prime \prime} & =g_{22} \tilde{\theta} \\
\tilde{\psi}^{\prime \prime} & =\left[g_{31} \tilde{\varphi}^{\prime}+g_{33} \tilde{\psi}^{\prime}\right] \theta^{\prime}
\end{aligned}
$$

Setting $\varphi^{\prime}=x_{1}$ and $\psi^{\prime}=x_{3}$ equations (16)a and (16)c become in matrix form:

$$
\left(\begin{array}{l}
x_{1}^{\prime} \\
x_{3}^{\prime}
\end{array}\right)=\theta^{\prime}\left(\begin{array}{ll}
g_{11} & g_{13} \\
g_{31} & g_{33}
\end{array}\right) \cdot\left(\begin{array}{l}
x_{1} \\
x_{3}
\end{array}\right)
$$

Let $\rho_{1,2}$ be the eigenvalues of system (17). Then:

$$
\rho_{1} \rho_{2}=\frac{4\left(\hat{I}_{\bar{z}}^{P}-1\right)\left(\hat{h} k_{1} \cot \theta+\hat{I}_{\bar{z}}^{P}+1\right)}{4\left(\hat{I}_{\bar{y}}^{P}-1\right) \hat{I}_{\bar{z}}^{P}-\hat{h}^{2} k_{1}^{2}} \theta^{\prime 2}=-\frac{6 k_{3}\left(2 \hat{h} k_{1} \cot \theta+k_{3}+4\right)}{\left[3 k_{3} k_{1}^{2}+k_{2}\left(k_{3}+2\right)\right] \hat{h}^{2}+3 k_{3}\left(k_{3}+2\right)} \theta^{\prime 2}<0
$$

Therefore, the system has two real distinct eigenvalues with one of those positive, which means that in any interval between two collisions of the drum with the horizontal plane, small out-of-plane perturbations of the motion grow exponentially in time (saddle point). Consequently, rocking is an unconditionally unstable motion, independently of the slenderness, $\hat{h}$, and the conicity, $\beta$, of the frustum. As a result, the three dimensional 
character of the problem prevails. This is not an astonishing result because rocking could be seen as an inverted pendulum motion. Pendulum motions are also extremely sensitive to out-of-plane perturbations as this is well-known. A numerical example confirms the above result from linear stability analysis; Consider the column depicted at Figure 4 . The column is a conical frustum with $h=4 m, r=0.4 m, R=0.5 m, \beta=0.8$ and $\hat{h}=8$. The equations of motion are integrated numerically for the following three cases of initial conditions:

$$
\begin{array}{lll}
\text { IC } 1: \varphi_{0}=0, & \theta_{0}=1^{\circ}, \quad \psi_{0}=0, & \varphi_{0}^{\prime}=0, \quad \theta_{0}^{\prime}=0, \quad \psi_{0}^{\prime}=0 \\
\text { IC2: } \varphi_{0}=0, \quad \theta_{0}=1^{\circ}, \quad \psi_{0}=0, \quad \varphi_{0}^{\prime}=0.01, \quad \theta_{0}^{\prime}=0, \quad \psi_{0}^{\prime}=0 \\
\text { IC3: } \varphi_{0}=0, \quad \theta_{0}=1^{\circ}, \quad \psi_{0}=0, \quad \varphi_{0}^{\prime}=0.1, \quad \theta_{0}^{\prime}=0, \quad \psi_{0}^{\prime}=0
\end{array}
$$

Initial condition IC1 refers to rocking. Notice that even for small values of $\varphi_{0}^{\prime}$ (IC2 and IC3) the contact point changes position (Figure 5) and it is not limited in the plane of the two dimensional rocking motion, where $\hat{X}(t)=0$ (IC1).

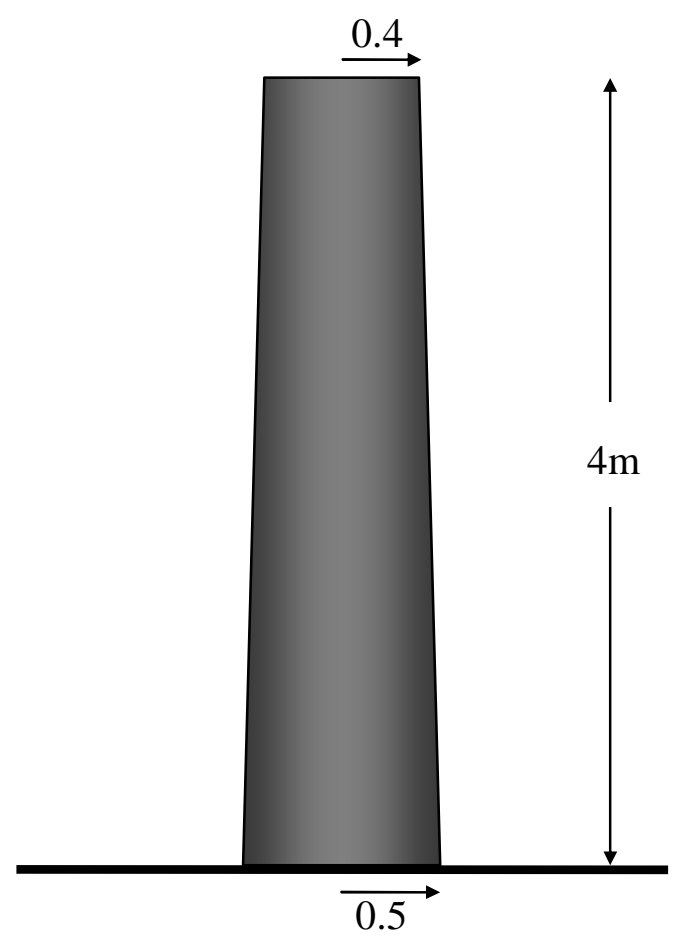

Figure 4. Geometry of the column considered for the numerical examples. 


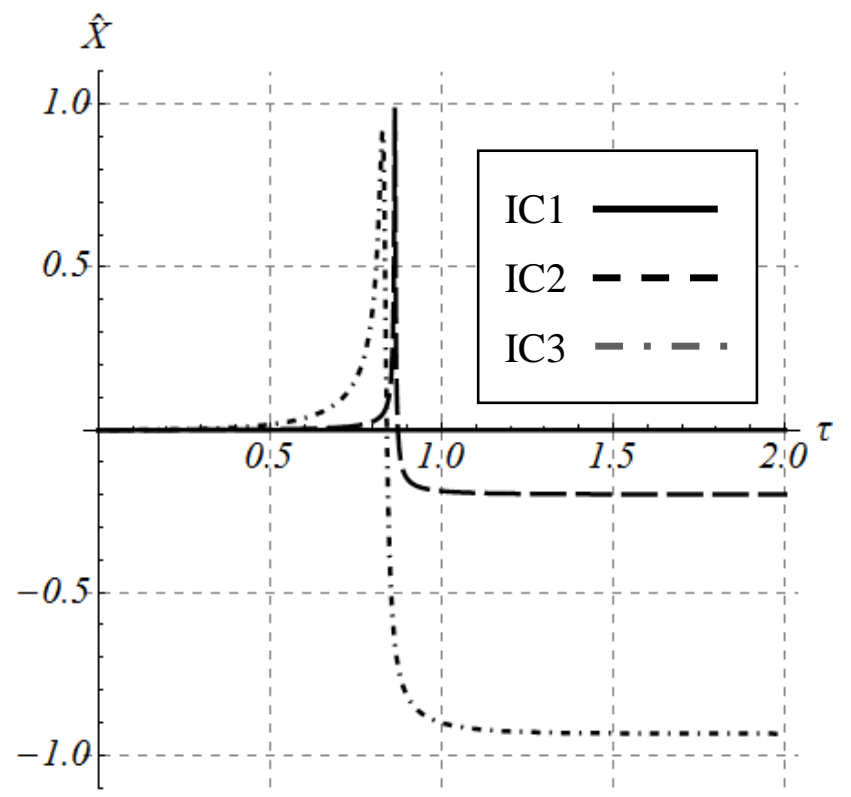

Figure 5. Coordinate, $\hat{X}$, of the contact point for initial conditions IC1, IC2 and IC3. Notice that for rocking (IC1) the $X_{P}$ coordinate of the drum remains constant (zero), while for IC2 and IC3 is not. This means that the contact point is not limited in the plane of the two dimensional rocking motion.

\subsection{Wobbling motion}

An animation of the above three dimensional motion reveals an interesting kind of motion that it is evolving for small inclination angles of the drum. In this paragraph, we distinguish this special kind of motion, which is not limited in the vertical plane of rocking, but it is rather evolving out-of-plane, in the three dimensional space, and is observed when the drum rolls on its edge under small inclination angles $(0<\theta<<1)$. We call this three dimensional motion for small inclination angles wobbling. Another example of this particular motion could also be a coin wobbling on a desk. The main difference of rocking from wobbling is that the pole of rotation of the body is not kept constant in time and that the motion of the frustum needs three angles to be described, i.e. the Euler angles, instead of one. Assuming small inclination angles, $\theta<<1$, Eqs. (12) are linearized as follows:

$$
\begin{aligned}
\varphi^{\prime \prime} & =\left[f_{11}(\theta) \varphi^{\prime}+f_{13}(\theta) \psi^{\prime}\right] \theta^{\prime}+f_{14}(\theta) \hat{u}_{g r}^{\prime \prime}+O(\theta) \\
\theta^{\prime \prime} & =\left[f_{21}(\theta) \varphi^{\prime}+f_{23}(\theta) \psi^{\prime}\right] \varphi^{\prime}+f_{22}(\theta)+f_{24}(\theta) \hat{u}_{g r}^{\prime \prime}+O\left(\theta^{2}\right) \\
\psi^{\prime \prime} & =\left[f_{31}(\theta) \varphi^{\prime}+f_{33}(\theta) \psi^{\prime}\right] \theta^{\prime}+f_{34}(\theta) \hat{u}_{g r}^{\prime \prime}+O(\theta) \\
\hat{X}^{\prime \prime} & =\varphi^{\prime} \psi^{\prime} \sin \varphi-\psi^{\prime \prime} \cos \varphi+\alpha_{1}^{g r} \hat{u}_{g r}^{\prime \prime} \\
\hat{Y}^{\prime \prime} & =-\varphi^{\prime} \psi^{\prime} \cos \varphi-\psi^{\prime \prime} \sin \varphi+\alpha_{2}^{g r} \hat{u}_{g r}^{\prime \prime}
\end{aligned}
$$

where:

$$
f_{11}=-\frac{1}{\theta}\left[\frac{4\left(\hat{I}_{\bar{z}}^{P}-1\right) \hat{I}_{\bar{z}}^{P}}{\hat{h}^{2} k_{1}^{2}-4\left(\hat{I}_{\bar{y}}^{P}-1\right) \hat{I}_{\bar{z}}^{P}}+2\right]+\frac{2 \hat{h} k_{1}\left(\hat{I}_{\bar{z}}^{P}-1\right)}{\hat{h}^{2} k_{1}^{2}-4\left(\hat{I}_{\bar{y}}^{P}-1\right) \hat{I}_{\bar{z}}^{P}} \quad ; \quad f_{13}=-\frac{1}{\theta} \frac{4 \hat{I}_{\bar{z}}^{P}\left(\hat{I}_{\bar{z}}^{P}-1\right)}{\hat{h}^{2} k_{1}^{2}-4\left(\hat{I}_{\bar{y}}^{P}-1\right) \hat{I}_{\bar{z}}^{P}}
$$




$$
\begin{aligned}
& f_{21}=\frac{2 \theta\left(\hat{I}_{\bar{y}}^{P}-\hat{I}_{\bar{z}}^{P}-1\right)-\hat{h} k_{1}}{2 \hat{I}_{\bar{y}}^{P}} ; f_{22}=\theta \frac{\hat{h} k_{1}}{2 \hat{I}_{\bar{y}}^{P}}-\frac{1}{\hat{I}_{\bar{y}}^{P}} \quad ; \quad f_{23}=-\frac{2 \theta \hat{I}_{\bar{z}}^{P}+\hat{h} k_{1}}{2 \hat{I}_{\bar{y}}^{P}} \\
& f_{31}=2 \frac{1}{\theta} \frac{2\left(-2 \hat{I}_{\bar{y}}^{P}+\hat{I}_{\bar{z}}^{P}+1\right) \hat{I}_{\bar{z}}^{P}+\hat{h}^{2} k_{1}^{2}}{\hat{h}^{2} k_{1}^{2}-4\left(\hat{I}_{\bar{y}}^{P}-1\right) \hat{I}_{\bar{z}}^{P}}-\frac{4 \hat{h}_{1} k_{1}\left(\hat{I}_{\bar{z}}^{P}-1\right)}{\hat{h}^{2} k_{1}^{2}-4\left(\hat{I}_{\bar{y}}^{P}-1\right) \hat{I}_{\bar{z}}^{P}} ; \\
& f_{33}=\frac{1}{\theta} \frac{4 \hat{I}_{\bar{z}}^{P}\left(\hat{I}_{\bar{z}}^{P}-1\right)}{\hat{h}^{2} k_{1}^{2}-4\left(\hat{I}_{\bar{y}}^{P}-1\right) \hat{I}_{\bar{z}}^{P}}-\frac{2 \hat{h}_{1}\left(\hat{I}_{\bar{z}}^{P}-1\right)}{\hat{h}^{2} k_{1}^{2}-4\left(\hat{I}_{\bar{y}}^{P}-1\right) \hat{I}_{\bar{z}}^{P}} \\
& f_{14}=-\frac{1}{\theta} \frac{2 \hat{h} k_{1}\left(\hat{I}_{\bar{z}}^{P}-1\right)}{4\left(\hat{I}_{\bar{y}}^{P}-1\right) \hat{I}_{\bar{z}}^{P}-\hat{h}^{2} k_{1}^{2}}\left(\alpha_{1}^{g r} \cos \varphi+\alpha_{2}^{g r} \sin \varphi\right) \quad ; \quad f_{24}=\left(\theta \frac{1}{\hat{I}_{\bar{y}}^{P}}+\frac{\hat{h} k_{1}}{2 \hat{I}_{\bar{y}}^{P}}\right)\left(-\alpha_{1}^{g r} \sin \varphi+\alpha_{2}^{g r} \cos \varphi\right) \\
& f_{34}=\left[\frac{4 \hat{I}_{\bar{y}}^{P}-\hat{h}^{2} k_{1}^{2}-4}{4\left(\hat{I}_{\bar{y}}^{P}-1\right) \hat{I}_{\bar{z}}^{P}-\hat{h}^{2} k_{1}^{2}}+\frac{1}{\theta} \frac{2 \hat{h}_{1}\left(\hat{I}_{\bar{z}}^{P}-1\right)}{4\left(\hat{I}_{\bar{y}}^{P}-1\right) \hat{I}_{\bar{z}}^{P}-\hat{h}^{2} k_{1}^{2}}\right]\left(\alpha_{1}^{g r} \cos \varphi+\alpha_{2}^{g r} \sin \varphi\right)
\end{aligned}
$$

It is worth mentioning that in the above linearization the approximation is not uniform, because if we truncate the $O(\theta)$ terms in Eq.(22)b an equilibrium point is lost.

\section{4 .FREE WOBBLING}

For zero ground accelerations we derive the rotational velocities of wobbling as functions of the inclination angle $\theta$ (see Appendix $\mathrm{C}$ ). These velocities are expressed as follows:

$$
\begin{aligned}
& \varphi^{\prime}=-c_{1} \frac{1}{p} e^{\frac{1}{s}}\left[\frac{1}{\theta}-\frac{s}{2} \frac{1}{\theta^{2}}\right]-c_{2} e^{\frac{-1}{s} \theta} \frac{1}{\theta^{2}} \\
& \psi^{\prime}=c_{1} \frac{1}{p} e^{\frac{1}{s} \theta}\left[-p+\frac{1}{\theta}-\frac{s}{2} \frac{1}{\theta^{2}}\right]+c_{2} e^{\frac{-1}{s} \theta} \frac{1}{\theta^{2}}
\end{aligned}
$$

where $p=\frac{2 \hat{h} k_{1}}{\hat{I}_{\bar{z}}^{P}}, s=\frac{4\left(\hat{I}_{\bar{y}}^{P}-1\right) \hat{I}_{\bar{z}}^{P}-\hat{h}^{2} k_{1}^{2}}{2 \hat{h} k_{1}\left(\hat{I}_{\bar{z}}^{P}-1\right)}$ and $c_{1}$ and $c_{2}$ are constants specified by the initial conditions:

$$
\begin{aligned}
& c_{1}=-e^{\frac{-\theta_{0}}{s}}\left(\varphi_{0}^{\prime}+\psi_{0}^{\prime}\right) \\
& c_{2}=e^{\frac{\theta_{0}}{s}}\left[\frac{2 \theta_{0}-s}{p}\left(\varphi_{0}^{\prime}+\psi_{0}^{\prime}\right)-\varphi_{0}^{\prime} \theta_{0}^{2}\right]
\end{aligned}
$$

Adding equations (23)a and (23)b yields to:

$$
\varphi^{\prime}+\psi^{\prime}=\left(\varphi_{0}^{\prime}+\psi_{0}^{\prime}\right) e^{\frac{\theta-\theta_{0}}{s}}
$$




\subsection{Approximations of rotational velocities}

The behavior of the drum for $\theta<\theta_{0}<<1$ is approximated by expanding Eqs.(23) in power series. Neglecting terms of $O\left(\frac{\theta}{\theta_{0}}\right)=O\left(\varepsilon^{1 / 2}\right)$ and $O\left(\frac{\theta_{0}^{3}}{\theta^{2}}\right)=O\left(\varepsilon^{1 / 2}\right)$ that is for $\theta_{0}=O\left(\varepsilon^{3 / 2}\right)$, $\theta=O\left(\varepsilon^{2}\right)$ and $0<\varepsilon<<1$, we obtain:

$$
\begin{aligned}
& \varphi^{\prime} \approx-\frac{2}{p s}\left(\varphi_{0}^{\prime}+\psi_{0}^{\prime}\right)+\frac{(p s+2) \varphi_{0}^{\prime}+2 \psi_{0}^{\prime}}{p s}\left(\frac{\theta_{0}}{\theta}\right)^{2}=O\left(\varepsilon^{-1}\right) \\
& \psi^{\prime} \approx \frac{p s+2}{p s}\left(\varphi_{0}^{\prime}+\psi_{0}^{\prime}\right)-\frac{(p s+2) \varphi_{0}^{\prime}+2 \psi_{0}^{\prime}}{p s}\left(\frac{\theta_{0}}{\theta}\right)^{2}=O\left(\varepsilon^{-1}\right)
\end{aligned}
$$

In equations (26) the second term on the right hand side is dominant and of $O\left(\varepsilon^{-1}\right)$ whereas the first term is a constant independent of $\varepsilon$. Equations (26) can be reduced to the approximation suggested by Srinivasan and Ruina [21] if one neglects the constant term and assumes that $\varphi_{0}^{\prime}$ and $\psi_{0}^{\prime}$ are quite small. This approximation is indeed satisfactory in the near collision state, but fails to reproduce the global response of the wobbling motion. The numerical integration of the equations of motion corroborates the current asymptotic approximations for $\varphi_{0}^{\prime}, \psi_{0}^{\prime} \neq 0$ and the comparison of the asymptotic with the fully numerical solution is presented in Figure 6 to Figure 8. For the numerical comparison, the same geometrical parameters were used with section 2. The initial values were: $\phi_{0}=0$, $\theta_{0}=\frac{\pi}{18} \approx 0.17, \psi_{0}=0, \phi_{0}^{\prime}=0.1, \theta_{0}^{\prime}=0, \psi_{0}^{\prime}=-0.2$; notice that the range of $\theta$ is within the limits of the above approximation.

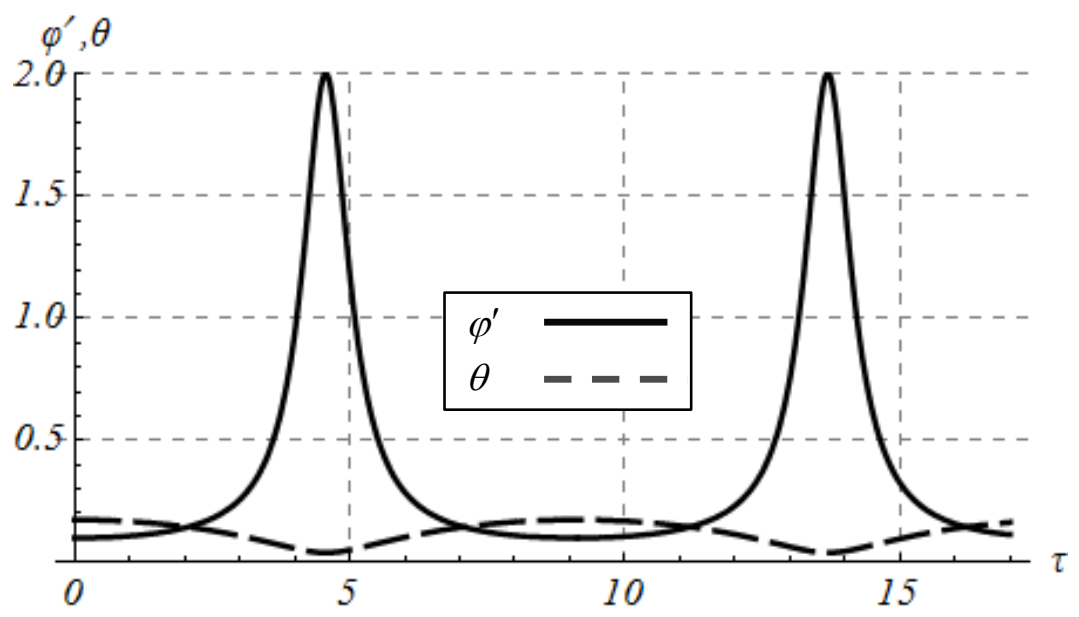

Figure 6. Precession velocity, $\varphi^{\prime}$, and inclination $\theta$ versus $\tau$. The precession velocity obtains large values for small inclination angles. 

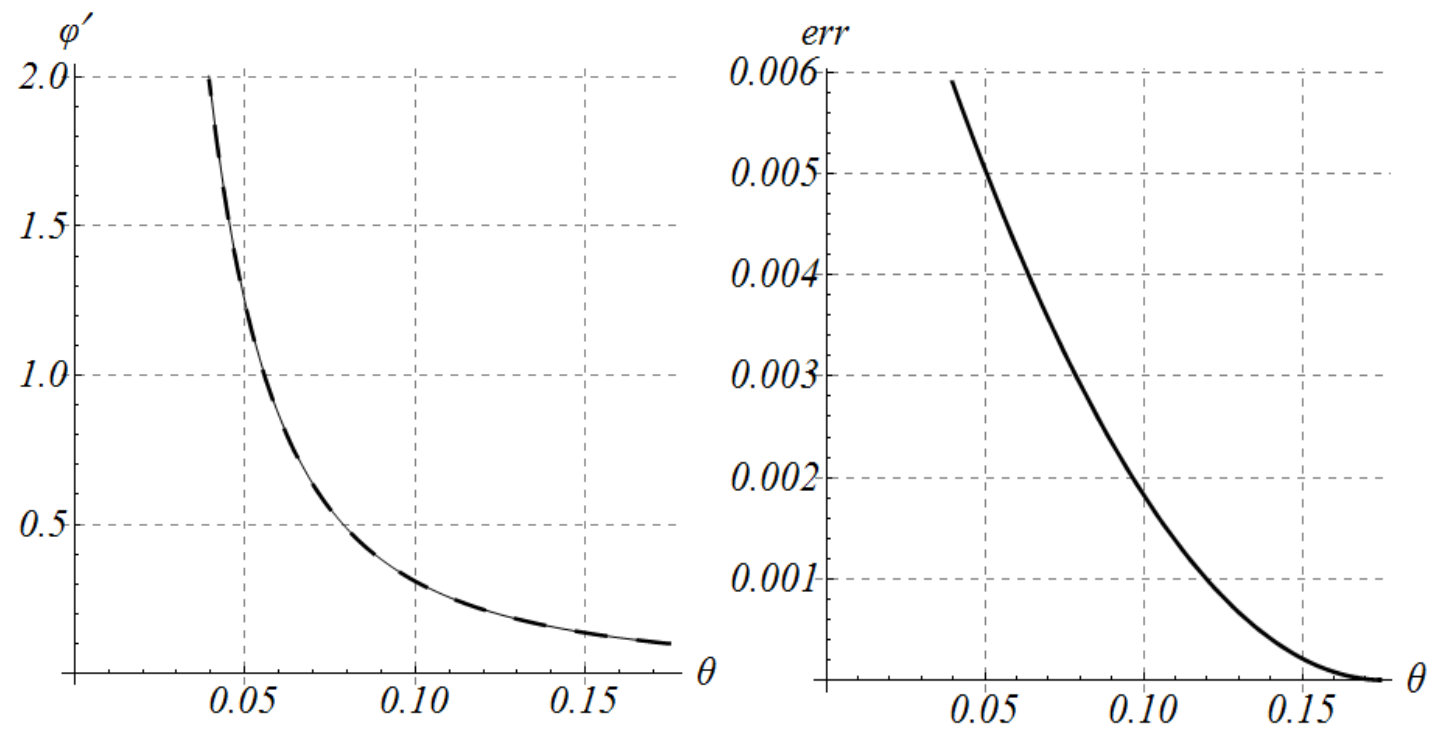

Figure 7. On the left $\varphi^{\prime}$ versus $\theta$ is plotted for a) the numerical solution (solid line) and $b$ ) the approximation of Eqs.(26) (dashed line). On the right the relative error of the approximation is presented, which is less than $6 \%$.
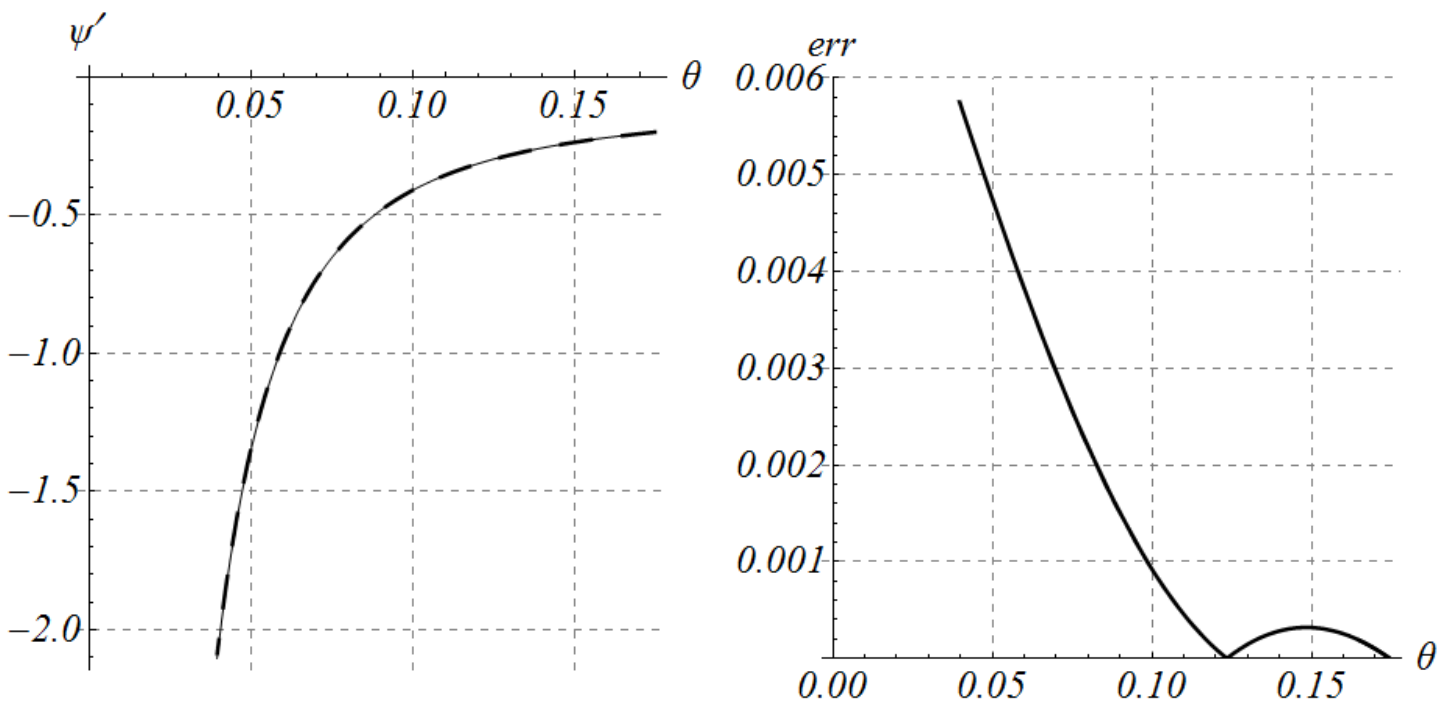

Figure 8. On the left $\psi^{\prime}$ versus $\theta$ is plotted for a) the numerical solution (solid line) and $b$ ) the approximation of Eqs.(26) (dashed line). On the right the relative error of the approximation is presented, which is less than 6\%.

Both angular velocities $\varphi^{\prime}$ and $\psi^{\prime}$ depend on the slenderness $\hat{h}$, and the conicity $\beta$ of the frustum because of the term $p s^{-1}$ in Eqs.(26). In Figure 9 we present the contour plot of $p s(\hat{h}, \beta)$. From the contours we observe that $\mathrm{ps}^{-1}$, and consequently $\varphi^{\prime}$ and $\psi^{\prime}$, increase for decreasing $\hat{h}$ and increasing $\beta$ with $\beta<0.5$. For $\beta>0.5$, the term $\mathrm{ps}^{-1}$ is practically not affected by $\beta$. This observation would support the approximation of typical classical column drums ( $\beta \approx 0.8$ ) by cylinders. However, this statement must be checked with numerical analysis of the multi-drum system response, which is out of the scope of the present paper. 


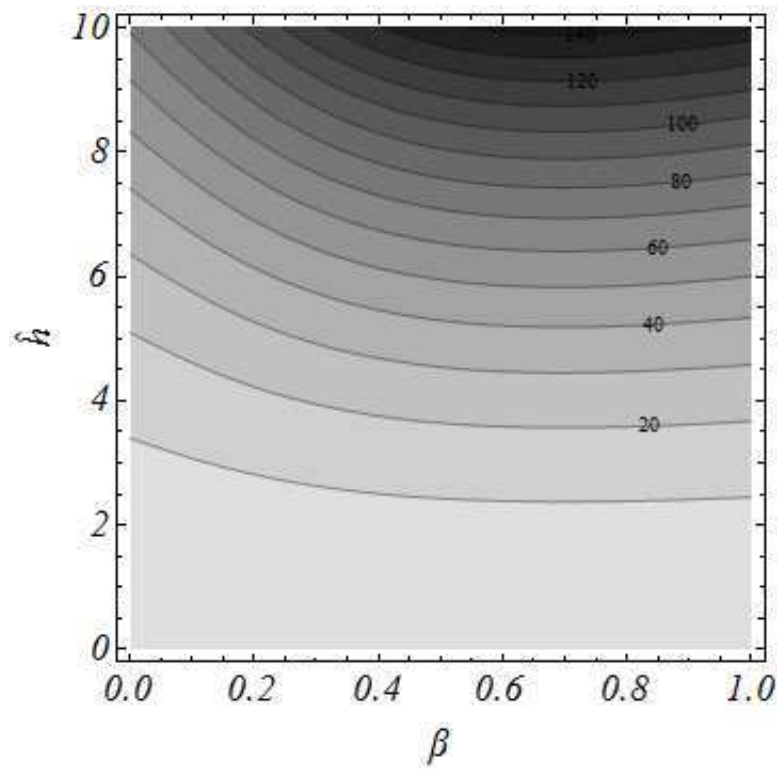

Figure 9. Contour plot of $p s(\hat{h}, \beta)$ as function of the slenderness $\hat{h}$ and the conicity $\beta$ of the conical frustum.

Equations (26), show that $\varphi^{\prime}$ and $\psi^{\prime}$, are quadratically dependent on the ratio $\frac{\theta_{0}}{\theta}$. Yet, their sum is practically constant (see Eq.(25)). Combining Eqs.(3) and (25), the spin of the drum is approximated by:

$$
\omega_{\zeta} \approx \varphi^{\prime}+\psi^{\prime} \approx \varphi_{0}^{\prime}+\psi_{0}^{\prime}
$$

From the above approximation we infer that for small inclination angles the spin of the frustum, $\omega_{\zeta}$, is defined by the initial conditions, it is independent of the geometrical parameters and it is practically constant in time. In Figure 10 we plot $\omega_{\zeta}(\tau)$ for:

$\phi_{0}=0, \quad \theta_{0}=\frac{\pi}{18}, \quad \psi_{0}=0, \phi_{0}^{\prime}=0.1, \quad \theta_{0}^{\prime}=0, \quad \psi_{0}^{\prime}=0.5$.

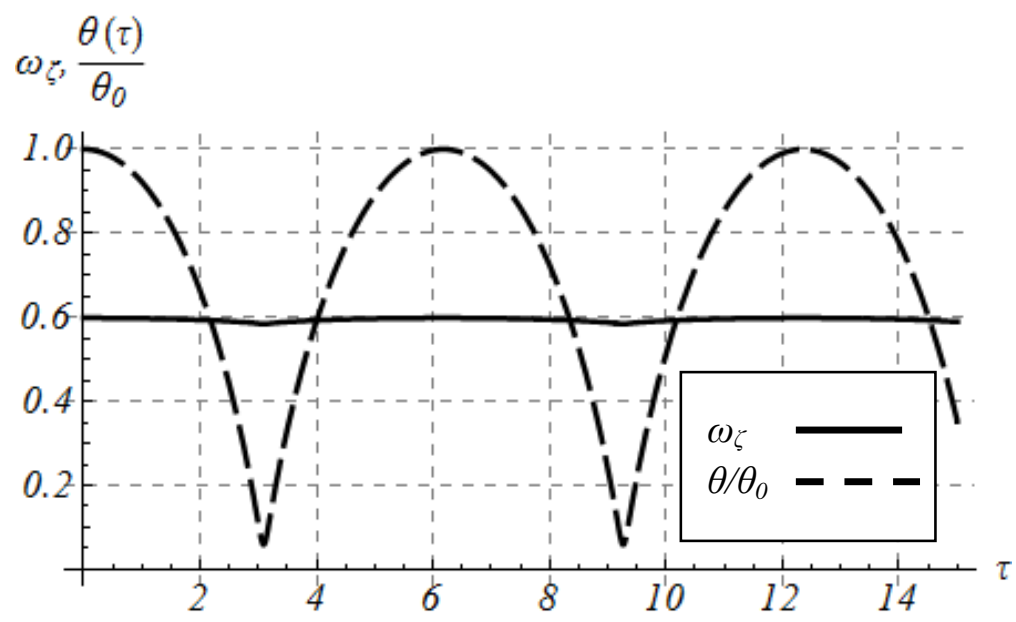

Figure 10. Spin, $\omega_{\zeta}$, of the body (solid line). It holds $\omega_{\zeta} \approx \varphi_{0}^{\prime}+\psi_{0}^{\prime}$. 


\subsection{Impact with the horizontal plane}

In the following we derive the condition for impact to take place. As mentioned above, at impact the drum is spinning, which shows that the dynamics of the collision are complex since they involve impulse reaction forces and torques, including torsion. This complication is pursued further here. Equations (23) show that the precession velocity and the spin increase and become infinite for $\theta \rightarrow 0^{+}$. Introducing Equations (23) into the total energy equation of the system, Eq.(13), we obtain:

$$
\hat{E}=\frac{\left(\hat{I}_{\bar{y}}^{P}-1\right)}{2 p^{2}}\left(\frac{s c_{1}-p c_{2}}{\theta}\right)^{2}+O\left(\frac{s c_{1}-p c_{2}}{\theta}\right)
$$

However, the total energy must be finite in the limit $\theta \rightarrow 0^{+}$. This implies that impact occurs only when the following condition is met:

$$
s c_{1}=p c_{2}
$$

Using Eqs.(24) the impact condition becomes:

$$
\left(\frac{\psi_{0}^{\prime}}{\varphi_{0}^{\prime}}\right)_{i m p}=\frac{p \theta_{0}^{2}}{\left(e^{\frac{2 \theta_{0}}{s}}-1\right) s+2 \theta_{0}}-1=\frac{p s}{2}-1+\frac{p}{3} \theta_{0}+\frac{p}{18 s} \theta_{0}^{2}+O\left(\theta_{0}^{3}\right)
$$

First we note that impact is not affected by the angular velocity $\theta_{0}^{\prime}$. By the same token, we note that the choice of the initial inclination angle, $\theta_{0}$, plays a subordinate role in the criterion for impact and in view of Eq.(27), we get from Eq.(30) that impact takes place if the (practically constant) spin of the frustum is:

$$
\omega_{\zeta} \approx \frac{p s}{2} \varphi_{0}^{\prime}
$$

Equation (31) shows that the impact condition is not affected by the conicity of the frustum, for $\beta>0.5$ (Figure 9). If the initial conditions satisfy exactly the impact condition, Eq.(29), the energy equation (13) can be solved for the inclination velocity at the instant of the impact, yielding to a non-zero value that is compatible with impact:

$$
\theta_{i m p}^{\prime}=-\sqrt{\frac{2 \hat{E}_{0}-\hat{I}_{\bar{z}}^{P} c_{1}^{2}+\hat{h} k_{1}}{\hat{I}_{\bar{y}}^{P}}} \neq 0
$$

On the contrary, if for given initial conditions the body does not overturn and the aforementioned impact condition is not met, the body oscillates between a maximum $\theta_{\max }$ and a minimum inclination angle $\theta_{\min }$ (Figure 11). The values of $\theta_{\max }$ and $\theta_{\min }$ can be specified by introducing Eqs.(23) into the total energy of the system, Eq.(13), leading to rather complicated algebraic expressions for $\theta_{\max , \min }$.

The fact that in the general case of initial conditions the conical drum does not collide with the horizontal plane is one more fundamental difference of the dynamics of rocking (2D) from wobbling (3D). 


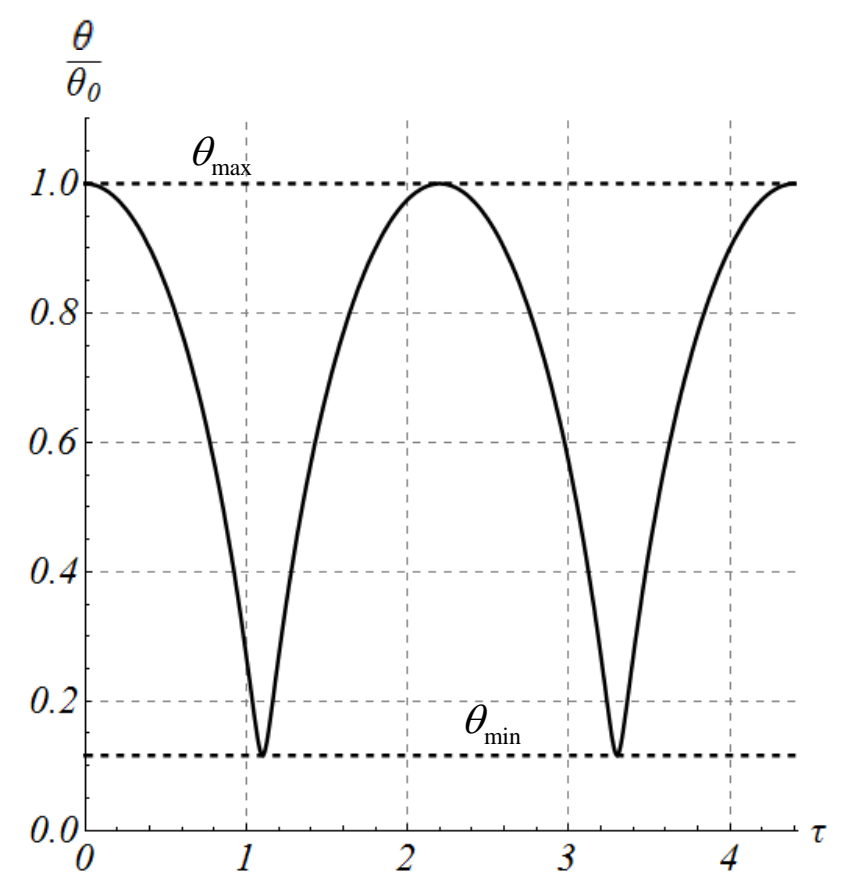

Figure 11. Oscillation of the conical frustum between $\theta_{\max }=\theta_{0}=\frac{\pi}{18}$ and $\theta_{\min }\left(\theta_{0}^{\prime}=0\right)$.

\section{APPLICATION TO ANCIENT CLASSICAL COLUMNS}

The last decades an increased interest arouse in the dynamic response of ancient classic and Hellenistic temples. In certain cases this kind of structures may undergo intense earthquake actions without collapsing. A particular element of these monuments is the multi-drum column. Each column is made by sometimes astoundingly fitted stone drums, which are placed without mortar on top of each other on a perfect fit [22]. From the mechanics point of view, the dynamic response of the classical columns, seen as rigid-body assemblies, is definitely non-linear and it involves rocking, sliding and wobbling of the drums (Figure 12). This response has also little in common with the dynamic response of modern structures, which exhibit 'tensegrity' (tension + integrity) in the sense that they can bear tensile stresses. The stability and resistance of modern columns to axial and lateral loads and moments is owed to the development of internal tensile forces, while the stability and resistance of classical columns is owed only to their self-weight [23] and geometric characteristics. This fundamental difference makes inapplicable most of the available structural theories and classical computational tools.

In the frame of a simplified mechanical model, both monolithic classical columns and single column drums can be seen as rigid conical frustums with slightly different radii. The vertical flutes that are often sculptured on the faces of the columns are of small extent and can be ignored in a first approach. Under these assumptions, the results that were exposed in the previous paragraphs can be used for the qualitative understanding of the dynamic behavior of a classical column. 


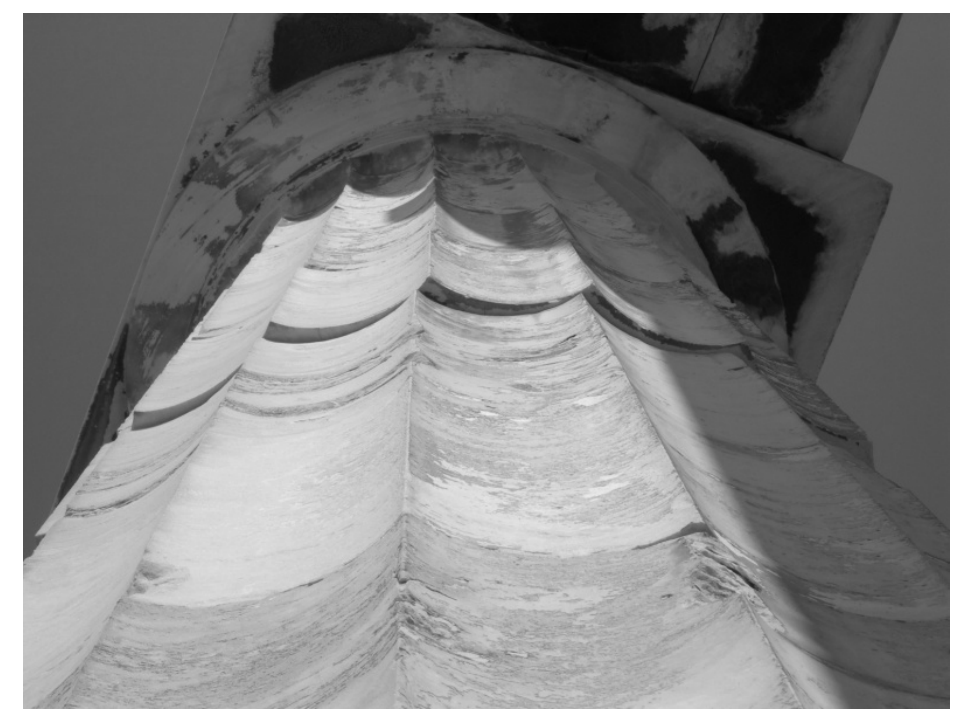

Figure 12. Mechanical damage of a column of Parthenon (see also Bouras et al. [24]). The relative displacements and rotations of the column drums corroborate the wobbling motion and the three dimensional dynamic response of these articulated systems.

According to Section 3, wobbling dominates rocking and therefore the drums of classical columns wobble under dynamic excitations. Consequently, the two dimensional analyses often performed [25, 26, 27, 28] should fail to capture the response of these articulated systems, as the out-of-plane motion cannot be ignored. In parallel, the mechanism of energy dissipation at wobbling is different from rocking. In particular, the dissipation of energy at wobbling is attributed to the frictional forces that are being developed at the joints during the stick and slip motion of the drums, while at rocking the dissipation is attributed to the impact with the horizontal plane. But even if impact is realized at the last moments of the wobbling motion, the spin of the drums involves frictional torsion at the collision, leading to an additional factor of energy dissipation. Figure 12, corroborates the above arguments, showing the relative rotations of the drums of a column of Parthenon.

The presented dynamic model can be used directly to study the dynamic behavior of classical monolithic columns ${ }^{3}$. However, for the study of multi-drum columns and colonnades, more sophisticated numerical tools have to be applied. The Distinct Element Method (DEM) seems to be a promising choice for the study of such systems and it has already been used for the modeling of multi-drum columns. The results were quite satisfactory [29]. However, the inherent theoretical assumptions of the above three dimensional numerical method that concern the contact laws, the contact detection and the integration of the equations of motion, question its reliability and accuracy for the modeling of such systems. The authors are not aware of any comparison of the above mentioned numerical method with the physical model proposed here (Eqs.12,19). However, this comparison exceeds the scope of the current paper and it should be followed in a different work.

\footnotetext{
${ }^{3}$ All the analytical calculations in the present paper have been performed with the symbolic language mathematical package Mathematica. The Mathematica files are available to the reader upon request to the corresponding author.
} 


\section{COCLUSIONS}

The objective of the present paper was the study of the wobbling motion of a conical frustum on a rough horizontal plane. For the analysis, the equations of motion were derived using Lagrangian formulation. The system is finally described by a set of non-linear equations that cover both the in-plane (rocking) and out-of-plane (wobbling) motion of the conical frustum. Linear stability analysis shows that rocking is unconditionally unstable and that wobbling is the dominant motion for frustums. In the general case of initial conditions, the frustum oscillates between a maximum and a minimum inclination angle. Impact takes place under certain initial conditions, while at the instant of the impact the spin of the frustum is not zero resulting to impact reaction forces and torques, including torsion. Practically, for small inclination angles, the spin of the body remains constant during the motion. On the contrary, large angular velocities appear as the inclination angle takes small values. The energy dissipation of the system is attributed to this instantaneous increase of the angular velocities, leading to a stick-slip motion of the drum, depending on the friction law. The aforementioned fundamental characteristics of the dynamic response of frustums enable us to get an insight to the dynamic behavior of classical multi-drum columns. In the particular case of single monolithic columns the proposed approach is directly applicable and may be treated as benchmark for other general purpose numerical tools, often used in the study of blocky systems.

\section{ACKNOWLEDGEMENTS}

The authors Ioannis Stefanou and Professor Anastasios Mavraganis would like to acknowledge the partial financial support of the present research by the Basic Research Program of National Technical University of Athens (NTUA), PEVE 2007.

\section{APPENDIX A}

\section{Matrices of the equations of motion $A$ and $B$}

$$
\mathbf{A}=\left(\begin{array}{ccccccc}
\hat{I}_{\bar{z}}^{P} \cos ^{2} \theta+\sin \theta\left(\hat{I}_{\bar{y}}^{P} \sin \theta-\sin \theta-\cos \theta \hat{h} k_{1}\right) & 0 & \cos \theta\left(\hat{I}_{\bar{z}}^{P}-1\right) & 0 & 0 & \frac{1}{2} f \cos \varphi & \frac{1}{2} f \sin \varphi \\
0 & \hat{I}_{\bar{y}}^{P} & 0 & 0 & 0 & \frac{1}{2} e \sin \varphi & -\frac{1}{2} e \cos \varphi \\
\cos \theta\left(\hat{I}_{\bar{z}}^{P}-1\right) & 0 & \hat{I}_{\bar{z}}^{P}-1 & -\cos \varphi & -\sin \varphi & 0 & 0 \\
\frac{1}{2} f \cos \varphi & \frac{1}{2} e \sin \varphi & 0 & -1 & 0 & 1 & 0 \\
\frac{1}{2} f \sin \varphi & -\frac{1}{2} e \cos \varphi & 0 & 0 & -1 & 0 & 1 \\
0 & 0 & \cos \varphi & 0 & 0 & 1 & 0 \\
0 & 0 & \sin \varphi & 0 & 0 & 0 & 1
\end{array}\right)
$$




$$
\mathbf{B}=\left(\begin{array}{c}
-\left(\hat{I}_{\bar{y}}^{P}-\hat{I}_{\bar{z}}^{P}-1\right) \sin 2 \theta \theta^{\prime} \varphi^{\prime}+\hat{h}_{1} \cos 2 \theta \theta^{\prime} \varphi^{\prime}+\sin \theta\left(\hat{I}_{\overline{z_{l}}}-1\right) \theta^{\prime} \psi^{\prime} \\
\frac{1}{2}\left(\sin 2 \theta\left(\hat{I}_{\bar{y}}^{P}-\hat{I}_{\bar{z}}^{P}-1\right) \varphi^{\prime 2}-\hat{h} k_{1} \cos 2 \theta \varphi^{\prime 2}-2 \sin \theta\left(\hat{I}_{\bar{z}}^{P}-1\right) \psi^{\prime} \varphi^{\prime}+f\right) \\
\sin \theta\left(\hat{I}_{\bar{z}}^{P}-1\right) \theta^{\prime} \varphi^{\prime} \\
\frac{1}{2}\left(f \theta^{\prime 2} \sin \varphi-2 e \varphi^{\prime} \theta^{\prime} \cos \varphi+f \varphi^{\prime 2} \sin \varphi\right) \\
-\frac{1}{2} f \theta^{\prime 2} \cos \varphi-e \varphi^{\prime} \theta^{\prime} \sin \varphi-\frac{1}{2} f \varphi^{\prime 2} \cos \varphi \\
\alpha_{1}^{g r} \hat{u}_{g r}^{\prime \prime}+\varphi^{\prime} \psi^{\prime} \sin \varphi \\
\alpha_{2}^{g r} \hat{u}_{\bar{g}}^{\prime \prime}-\varphi^{\prime} \psi^{\prime} \cos \varphi
\end{array}\right)
$$

where $e=2 \sin \theta+\hat{h} k_{1} \cos \theta$ and $f=\frac{d e}{d \theta}=\hat{h} k_{1} \sin \theta-2 \cos \theta$.

\section{APPENDIX B}

\section{Validation of the numerical integration scheme}

As was first proposed by Appel [6] and Korteweg [7], Eqs.(12) can be transformed as follows:

$$
\begin{gathered}
\frac{d^{2} \omega_{\zeta}}{d \theta^{2}}+\cot \theta \frac{d \omega_{\zeta}}{d \theta}-\frac{e}{\sin \theta} \frac{2\left(\hat{I}_{\bar{z}}^{P}-1\right)}{\hat{h}^{2} k_{1}^{2}-4\left(\hat{I}_{\bar{y}}^{P}-1\right) \hat{I}_{\bar{z}}^{P}} \omega_{\zeta}=0 \\
\omega_{\eta}=\frac{\hat{h}^{2} k_{1}^{2}-4\left(\hat{I}_{\bar{y}}^{P}-1\right) \hat{I}_{\bar{z}}^{P}}{\hat{h}^{2} k_{1}^{2}-4 \hat{I}_{\bar{y}}^{P}+4} \frac{d \omega_{\zeta}}{d \theta}+\frac{2 \hat{h} k_{1}\left(\hat{I}_{\bar{z}}^{P}-1\right)}{\hat{h}^{2} k_{1}^{2}-4 \hat{I}_{\bar{y}}^{P}+4} \omega_{\zeta}
\end{gathered}
$$

In the case of an infinite thickness disk ( $\hat{h}=0$ and $\left.k_{1}=k_{2}=k_{3}=\beta=1\right)$ Eq. (B.1) reduces to the one derived by Appell. Setting $q=\cot \theta$, Equation (B.1) yields:

$$
\left(q^{2}+1\right)^{2} \frac{d^{2} \omega_{\zeta}}{d s^{2}}+q\left(1+q^{2}\right) \frac{d \omega_{\zeta}}{d s}+4(b+c q) \omega_{\zeta}=0
$$

where $b=\frac{\hat{I}_{\bar{z}}^{P}-1}{\hat{h}^{2} k_{1}^{2}-4\left(\hat{I}_{\bar{y}}^{P}-1\right) \hat{I}_{\bar{z}}^{P}}$ and $c=\frac{1}{2} \hat{h} k_{1} \frac{\hat{I}_{\bar{z}}^{P}-1}{\hat{h}^{2} k_{1}^{2}-4\left(\hat{I}_{\bar{y}}^{P}-1\right) \hat{I}_{\bar{z}}^{P}}$.

Equation (B.3) is a special case of the Riemann-Papperitz equation with two singular points. Its solution is given in terms of hypergeometric functions below (Batista, [17]):

$$
\omega_{\zeta}(\theta)=A_{1} T_{\Delta}(\theta)+A_{2} T_{-\Delta}(\theta)
$$

where:

$A_{1}, A_{2}$ are complex constants that depend on the initial conditions of $\omega_{\zeta}$ and its derivative at $\theta=\theta_{0}, T_{\Delta}(\theta)=\left(\frac{q-i}{q+i}\right)^{\frac{1}{4}+\frac{\Delta}{2}} F\left(\frac{1+\Delta+\Delta^{*}}{2}, \frac{1+\Delta-\Delta^{*}}{2} ; 1+\Delta ; \frac{q-i}{q+i}\right), \Delta=\sqrt{\frac{1}{4}+4(b+i c)}$, 
$\Delta^{*}=\sqrt{\frac{1}{4}+4(b-i c)}$ and $F(a, b ; c ; z)$ is the Gauss hypergeometric function (Abramowitz and Stegun [30], 1970).

Combining Eqs.(13), (B.2) and (B.4) the equations of motion are integrated for given initial conditions. The integration involves the calculation of the Gauss Hypergeometric function, which finally is performed numerically. For that reason, the result is obtained through a semianalytical approach.

The validity of the numerical integration scheme that was currently applied was juxtaposed with the abovementioned semi-analytical solution. The solutions obtained were identical, with an average relative error, $\frac{\omega_{\eta}^{a n}-\omega_{\eta}^{n u m}}{\omega_{\eta}^{a n}}$, of order of magnitude $10^{-8}$.

\section{APPENDIX C}

\section{Analytical solution of the wobbling ODEs}

Equations (22)a and (22)c are linear in respect of $\varphi^{\prime}$ and $\psi^{\prime}$ and are written in matrix form:

$$
\mathbf{X}^{\prime}=\mathbf{L} \mathbf{X}
$$

where

$$
\begin{aligned}
& \mathbf{X}=\left(\begin{array}{c}
\varphi^{\prime} \\
\psi^{\prime}
\end{array}\right) \text { and } \\
& \mathbf{L}=\frac{1}{3} \frac{\theta^{\prime}}{\theta}\left(\begin{array}{cc}
-2 \frac{6 \hat{I}_{\bar{z}}^{P 2}-\left(12 \hat{I}_{\bar{y}}^{P}+3 \hat{h} k_{1} \theta-6\right) \hat{I}_{\bar{z}}^{P}+\hat{h} k_{1}\left(3 \hat{h} k_{1}+3 \theta\right)}{4\left(\hat{I}_{\bar{y}}^{P}-1\right) \hat{I}_{\bar{z}}^{P}-\hat{h}^{2} k_{1}^{2}} & \frac{12\left(\hat{I}_{\bar{z}}^{P}-1\right) \hat{I}_{\bar{z}}^{P}}{4\left(\hat{I}_{\bar{y}}^{P}-1\right) \hat{I}_{\bar{z}}^{P}-\hat{h}^{2} k_{1}^{2}} \\
-\frac{12 \hat{I}_{\bar{z}}^{P 2}+2\left(-12 \hat{I}_{\bar{y}}^{P}-6 \hat{h} k_{1} \theta+6\right) \hat{I}_{\bar{z}}^{P}+6 \hat{h}^{2} k_{1}^{2}+12 \hat{h} k_{1} \theta}{4\left(\hat{I}_{\bar{y}}^{P}-1\right) \hat{I}_{\bar{z}}^{P}-\hat{h}^{2} k_{1}^{2}} & \frac{2\left(\hat{I}_{\bar{z}}^{P}-1\right)\left(3 \hat{h} k_{1} \theta-6 \hat{I}_{\bar{z}}^{P}\right)}{4\left(\hat{I}_{\bar{y}}^{P}-1\right) \hat{I}_{\bar{z}}^{P}-\hat{h}^{2} k_{1}^{2}}
\end{array}\right)
\end{aligned}
$$

The eigenvalues and eigenvectors of $\mathbf{L}$ are:

$$
\begin{aligned}
& \lambda_{1}=\frac{2 \hat{h} k_{1}\left(\hat{I}_{\bar{z}}^{P}-1\right) \theta^{\prime}}{4\left(\hat{I}_{\bar{y}}^{P}-1\right) \hat{I}_{\bar{z}}^{P}-\hat{h}^{2} k_{1}^{2}}, \quad \lambda_{2}=\left(\frac{2 \hat{h} k_{1}\left(\hat{I}_{\bar{z}}^{P}-1\right)}{\hat{h}^{2} k_{1}^{2}-4\left(\hat{I}_{\bar{y}}^{P}-1\right) \hat{I}_{\bar{z}}^{P}}-\frac{2}{\theta}\right) \theta^{\prime} \\
& \mathbf{S}=\left(\begin{array}{cc}
-\frac{2 \hat{I}_{\bar{z}}^{P}\left(\hat{I}_{\bar{z}}^{P}-1\right)}{2\left(-2 \hat{I}_{\bar{y}}^{P}+\hat{I}_{\bar{z}}^{P}-\theta \hat{h} k_{1}+1\right) \hat{I}_{\bar{z}}^{P}+\hat{h} k_{1}\left(2 \theta+\hat{h} k_{1}\right)} & -1 \\
1 & 1
\end{array}\right)
\end{aligned}
$$

with

$$
\mathbf{X}=\mathbf{S} \mathbf{Y}
$$

Replacing (C.3) into (C.1) and after some algebra we obtain:

$$
\mathbf{Y}^{\prime}=\left(\mathbf{D}-\mathbf{S}^{-1} \mathbf{S}^{\prime}\right) \mathbf{Y}
$$


where $\mathbf{D}=\left(\begin{array}{cc}\lambda_{1} & 0 \\ 0 & \lambda_{2}\end{array}\right)$.

Equation (C.4) can be solved for $\mathbf{Y}$ and the solution of (C.1) is obtained using Eq.(C.3):

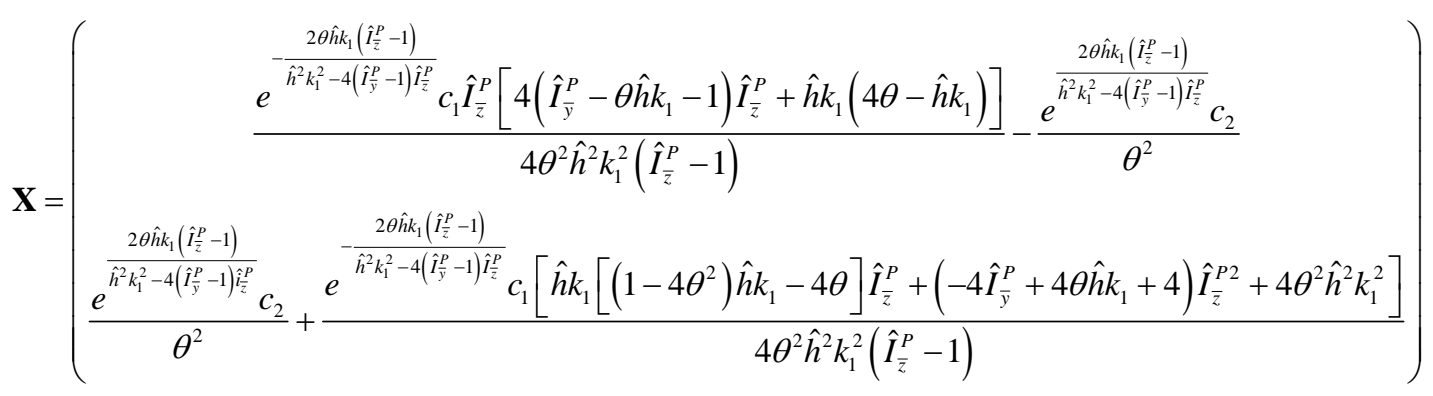

\section{REFERENCES}

[1] E.J. Routh, An elementary treatise on the dynamics of a system of rigid bodies, MacMillan, 1868.

[2] E.J. Routh, Advanced part of a treatise on the dynamics of a system of rigid bodies, MacMillan, 1905.

[3] A.G. Webster, The dynamics of particles and of rigid, elastic and fluid bodies, second ed., Leipzig, 1925.

[4] P. Appell, Traite de mécanique rationnelle, Gauthier-Villars, Paris, 1931.

[5] W.D. MacMillan, Dynamics of rigid bodies, Dover Publications, New York, 1936.

[6] P. Appell, Sur l'intégration des équations de mouvement d'un corps pesant de révolution roulant par une arrête circulaire sur un plan horizontal; cas particulier du cerceau, Rend. Circ. Mat. Palermo. 14 (1900), 1-6.

[7] D. Korteweg, Extrait d'une lettre a M. Appell, Rend. Circ. Mat. Palermo. 14 (1900), 7-8.

[8] E.G. Gallop, On the rise of a spinning top, Trans. Camb. Phil. Soc. 19 (1904), 356-373.

[9] O.M. O'Reilly, The dynamics of rolling discs and sliding discs, Nonlinear Dynam. 10 (1996), 287-385.

[10] A.S. Kuleshov, The Steady Rolling of a Disc on a Rough Plane, J. Appl. Math. Mech. 65 (2001), 171-173.

[11] P.C. Paris, L. Zhang, A disc rolling on a horizontal surface without slip, Math. Comput. Model. 36 (2002), 855-860.

[12] P. Kessler, O.M. O'Reilly, The Ringing of Euler's Disc, Regul. Chaotic Dyn. 7 (2002), 49-60.

[13] A.V. Borisov, I.S. Mamaev, A.A. Kilin, Dynamics of rolling disc, Regul. Chaotic Dyn. 7 (2003), 201-212.

[14] C. Le Saux, R.I. Leine, C. Glocker, Dynamics of a Rolling Disc in the Presence of Dry Friction, J. Nonlinear Sci. 15 (2005), 27-61.

[15] A.S. Koh, G. Mustafa, Free rocking of cylindrical structures, J. Eng. Mech.-A.S.C.E. 116 (1990), 35-54. 
[16] M. Batista, Steady motion of a rigid disc of finite thickness on a horizontal plane, Int. J. Nonlin. Mech. 41 (2006), 605-621.

[17] M. Batista, Integrability of the motion of a rolling disc of finite thickness on a plane, Int. J. Nonlin. Mech. 41 (2006), 850-859.

[18] M. Batista, The nearly horizontally rolling of a thick disk on a rough plane, Regul. Chaotic Dyn. 13 (2008), 344-354.

[19] J.R. Rice, S.T. Tse, Dynamic Motion of a Single Degree of Freedom System Following a Rate and State Dependent Friction Law, J. Geophys. Res.-Solid. 91 (1986), 521-530.

[20] R. Rosenberg, Analytical Dynamics of Discrete Systems,Plenum Press, New York, 1977.

[21] M. Srinivasan, A. Ruina, Rocking and rolling: A can that appears to rock might actually roll, Phys. Rev. E. 78 (2008).

[22] M. Korres, G.A. Panetsos, T. Seki, The Parthenon Architecture and Conservation, Hellenic Foundation for Culture, Athens, 1999.

[23] D. Konstantinidis, N. Makris, The rocking spectrum and the limitations of practical design methodologies, Earthquake Eng. Struc. 32 (2003), 265-289.

[24] M. Bouras, K. Zambas, S. Mavrommatis, The Works of the Committee for the Preservation of the Acropolis Monuments on the Acropolis of Athens, Hellenic Ministry, Athens, Greece, 2002.

[25] I.N. Psycharis, D.Y. Papastamatiou, A.P. Alexandris, Parametric investigation of the stability of classical columns under harmonic and earthquake excitations. Earthquake Eng. Struc. 29 (2000), 1093-1109.

[26] D. Konstantinidis, N. Makris, Seismic response analysis of multisrum classical columns, Earthquake Eng. Struc. 34 (2005), 1243-1270.

[27] G. Yagoda, Y. Hatzor, Seismic Risk Estimation from Overturning Analysis of Hellenistic Columns using DDA, in: 8th International Conference on Analysis of Discontinuous Deformation (ICADD-8). Beijing, China, 2007.

[28] P. Komodromos, L. Papaloizou, P. Polycarpou, Simulation of the response of ancient columns under harmonic and earthquake excitations. Eng. Struct. 30 (2008), 2154-2164.

[29] C. Papantonopoulos, I.N. Psycharis, D.Y. Papastamatiou, J.V. Lemos, H.P. Mouzakis, Numerical prediction of the earthquake response of classical columns using the distinct element method. Earthquake Eng. Struc. 31 (2002), 1699-1717.

[30] M. Abramowitz, I.A. Stegun, Handbook of Mathematical Functions, with Formulas, Graphs and Mathematical Tables, Dover Publications, 1970. 


\section{LEGENDS}

Figure 1.The conical frustum: 3D and 2D view.

Figure 2. Coordinate systems and Euler angles. $\mathrm{O}(\mathrm{X}, \mathrm{Y}, \mathrm{Z})$ is the inertial frame, $\mathrm{P}\left(\mathrm{X}_{\mathrm{P}}, \mathrm{Y}_{\mathrm{P}}\right)$ the contact point with the horizontal plane, $\theta$ the inclination angle, $\varphi$ the precession angle and $\psi$ the rotation about $\zeta$-axis.

Figure 3. Mobilized friction coefficient and normalized inclination angle of the column presented in Figure 4. The energy dissipated during the short time intervals of sliding (peaks of the mobilized friction coefficient) generally results to a practically smooth collisionless contact.

Figure 4. Geometry of the column considered for the numerical examples.

Figure 5. Coordinate, $\hat{X}$, of the contact point for initial conditions IC1, IC2 and IC3. Notice that for rocking (IC1) the $\mathrm{X}_{\mathrm{P}}$ coordinate of the drum remains constant (zero), while for IC2 and IC3 is not. This means that the contact point is not limited in the plane of the two dimensional rocking motion.

Figure 6. Precession velocity, $\varphi^{\prime}$, and inclination $\theta$ versus $\tau$. The precession velocity obtains large values for small inclination angles.

Figure 7. On the left $\varphi^{\prime}$ versus $\theta$ is plotted for a) the numerical solution (solid line) and b) the approximation of Eqs.(26) (dashed line). On the right the relative error of the approximation is presented, which is less than $6 \%$.

Figure 8. On the left $\psi^{\prime}$ versus $\theta$ is plotted for a) the numerical solution (solid line) and b) the approximation of Eqs.(26) (dashed line). On the right the relative error of the approximation is presented, which is less than $6 \%$.

Figure 9. Contour plot of $p s(\hat{h}, \beta)$ as function of the slenderness $\hat{h}$ and the conicity $\beta$ of the conical frustum.

Figure 10. Spin, $\omega_{\zeta}$, of the body (solid line). It holds $\omega_{\zeta} \approx \varphi_{0}^{\prime}+\psi_{0}^{\prime}$.

Figure 11. Oscillation of the conical frustum between $\theta_{\max }=\theta_{0}=\frac{\pi}{18}$ and $\theta_{\min }, \theta_{0}^{\prime}=0$.

Figure 12. Mechanical damage of a column of Parthenon (see also Bouras et al. [24]). The relative displacements and rotations of the column drums corroborate the wobbling motion and the three dimensional dynamic response of these articulated systems. 